\title{
Validación de un instrumento para medir la competencia digital de estudiantes universitarios (CODIEU)
}

\section{Validation of an instrument to measure the digital competence of university students (CODIEU)}

\author{
Marcos Cabezas*, Sonia Casillas*, Manuela Ferreira**, Fernando Teixeira** \\ *Universidad de Salamanca, España, **Universidad de Oporto, Portugal
}

\begin{abstract}
Resumen
Para que la integración de las TIC en las aulas universitarias contribuya al desarrollo de procesos pedagógicos de calidad, es necesario que los estudiantes posean un adecuado nivel de competencia digital. Para investigar esta competencia, necesitamos de instrumentos válidos y fiables que permitan recoger información sobre la misma. El principal objetivo es el de identificar las propiedades psicométricas de un cuestionario para medir la competencia digital de estudiantes universitarios (CODIEU), a partir de la autoevaluación que realizan los mismos, de acuerdo a tres constructos: conocimientos sobre TIC; manejo de dispositivos, herramientas y servicios TIC; y actitudes hacia las mismas.

Palabras clave: cuestionario, competencia digital, tecnología de la educación, enseñanza superior.
\end{abstract}

\begin{abstract}
In order for the integration of ICT in university classrooms to contribute to the development of quality pedagogical processes, students must have an adequate level of digital competence. In order to investigate this competence, we need valid and reliable instruments to collect information about it. The main objective of this work is to identify the psychometric properties of a questionnaire to measure the digital competence of university students (CODIEU), based on their self-evaluation, according to three constructs: knowledge about ICT; handling of ICT devices, tools and services; and their attitudes towards them.

Keywords: questionnaire, digital competence, educational technology, higher education.
\end{abstract}

\section{Fundamentación teórica}

Con la implantación del Espacio Europeo de Educación Superior (EEES), uno de los grandes retos es el de poner en práctica un modelo docente centrado más en el de aprendizaje y en el desarrollo de competencias, algo que debe de superar la adquisición, por parte de los alumnos, de una multitud de conocimientos de la que no encuentren una utilidad más allá de su paso por la Universidad (García y Morillas, 2011; Gavari, 2006). Las Tecnologías de la Información y la Comunicación (TIC) pueden flexibilizar los procesos de enseñanzaaprendizaje para contribuir a la transformación de la
Universidad en una sociedad del conocimiento y el aprendizaje (Mirete, García y Hernández, 2015).

Para que la integración de las TIC en las aulas universitarias contribuya al desarrollo de procesos pedagógicos de calidad, es necesario que los estudiantes posean un adecuado nivel de competencia digital, entendida esta como: "el uso seguro y crítico de las tecnologías de la sociedad de la información (TSI) para el trabajo, el ocio y la comunicación. Se sustenta en las competencias básicas en materia de TIC: el uso de ordenadores para obtener, evaluar, almacenar, producir, presentar e intercambiar información, y comunicarse y participar en redes de colaboración a través de Internet." (Unión Europea, 2007, 7). Se trata de la capacidad para adquirir los conocimientos necesarios para manejarse en la sociedad del conocimiento y ser capaces de utilizar adecuadamente las TIC para buscar, evaluar, seleccionar, gestionar y comunicar la información en cualquier ámbito de especialización (CRUE y REBIUN, 2012).

Para investigar la competencia digital de los alumnos universitarios, necesitamos de instrumentos válidos y fiables que permitan realizar investigaciones sobre este tema. Realizada una revisión al respecto, son numerosos los estudios que utilizan cuestionarios para medir la percepción o autoevaluación de profesores y alumnos sobre sus competencias digitales, realizadas en distintos niveles educativos, desde perspectivas conceptuales, procedimentales y actitudinales (Cabezas, Casillas y Pinto, 2014; Centeno y Cubo, 2013; Fernández, Fernández y Cebreiro, 2016; Flores y Roig, 2016; Martín, Sáenz, Santiago y Chocarro, 2016; Roblizo, Sánchez y Cózar, 2015; Torres y Vidal, 2015). Por ello consideramos útil poder contar con un cuestionario, válido y fiable, que permita recoger una información, lo más completa posible, sobre las dimensiones de conocimiento, manejo y actitud que tienen los estudiantes universitarios hacia las TIC.

\section{Método}

El principal objetivo que se pretende conseguir es el de identificar las propiedades psicométricas de un cuestionario para medir el nivel de formación en 
competencia digital de alumnos universitarios (CODIEU).

La muestra piloto seleccionada para la validación del cuestionario está formada por 656 estudiantes de la Facultad de Educación de la Universidad de Salamanca y de la Universidad de Oporto, de los Grados de Educación Infantil, Educación Primaria y Educación Social; de los cuales el $20 \%$ son hombres y el $80 \%$ son mujeres, con edades comprendidas entre los 18 y los 24 años.

En la fase dedicada a la elaboración del cuestionario, en un primer momento se confeccionó una primera batería de preguntas sobre las dimensiones: a) Conocimiento, b) Manejo: dispositivos, herramientas y servicios, c) Actitud. Esta batería se sometió a un grupo de discusión de personas especialistas resultando una primera versión del cuestionario. El instrumento está compuesto por 90 ítems, cinco de los cuales son variables de identificación. Después de una primera depuración los ítems fueron reducidos a 88 reactivos. Se eligió una escala ordinal con valoración tipo Likert de 0 a 10 , siendo 0 el valor mínimo y 10 el máximo. La puntuación media (5) es la que indica si el estudiante se considera capaz (apto) para realizar las acciones que se le proponen.

Respecto a la validez del contenido, el cuestionario inicial se sometió a juicio de expertos, todos ellos profesionales especialistas en la materia objeto de estudio, incluyendo a profesionales de diferentes países europeos (Cubo, Martín, y García, 2011). Se tuvieron en consideración todas las aportaciones realizadas por los expertos.

Para el análisis de los datos se empleó el programa estadístico informático Statistical Package for the Social Sciences (SPSS v.21). Fueron llevados a cabo los siguientes análisis: (1) fiabilidad mediante el alfa de Cronbach, tanto para la totalidad de la escala como para cada una de las dimensiones que la componen, fundamentada especialmente en la covariación de los ítems; (2) índice de adecuación muestral KMO y prueba de esfericidad de Bartlett para la totalidad de la escala, como análisis previo al proceso de factorización, lo que permitió comprobar que los datos se correlacionaban positivamente, para poder llevar a cabo la (3) validez, por medio de un análisis factorial con el método de rotación varivax con normalización Kaiser, estudiando los componentes principales de cada uno de los ítems que conforman las tres dimensiones establecidas (conocimiento, manejo, actitud).

\section{Resultados}

Entre los principales resultados, cabe destacar que, se efectuó un análisis de validez de constructo. Para ello se realizó un análisis factorial mediante el estudio de los componentes principales con el método de rotación varivax con normalización Kaiser. Asimismo, la técnica de análisis factorial utilizada fue la multivariante, más comúnmente empleada en el área de Ciencias Sociales. En concreto, si nos centramos en el análisis de componentes principales vemos que su pretensión es la de comprobar la correlación entre variables para realizar una reducción de los datos a partir de simplificar la estructura.

El análisis factorial es una prueba que alcanza gran relevancia para la investigación. Por medio de la misma se comprueba la unidimensionalidad de las variables. Tras realizar un análisis factorial exploratorio, forzando el número de factores a 3 , finalmente se alcanza la unidimensionalidad con un tanto por ciento elevado de varianza explicada $(55,79 \%)$ y se confirma, en este sentido, la validez de la prueba.

La dimensión sobre conocimientos en TIC explica el $29,73 \%$ de la varianza total, la de manejo de TIC un $13,43 \%$ de la varianza total, en las tres subdimensiones contempladas (dispositivos, herramientas y servicios), y la de actitud un $12,62 \%$ de la varianza total. Todas ellas con un peso factorial alto en la totalidad de la escala.

El análisis de componentes principales realizado con la dimensión conocimiento sobre TIC se ha forzado a tres factores o dimensiones. En el primero de ellos saturan los ítems $(<0.40)$ : Sé lo que es la e-exclusión, la e-inclusión, el b-learning, el m-learning, la e-participación, un MOOC, el empoderamiento, la sindicación, el copyleft, la Web 3.0, el e-learning, un marcador social, la brecha digital, un mashup, la Web 2.0, una licencia Creative Commons y las Tecnologías para el Empoderamiento y la Participación (TEP), Sé lo que es la Realidad Virtual (RV), el ciberactivismo, la alfabetización digital, la blogosfera, la Realidad Aumentada (RA), el almacenamiento en la nube, un Objeto de Aprendizaje (OA) y las Tecnologías para el Aprendizaje y el Conocimiento (TAC). Con estos resultados se puede determinar que este factor incluye ítems por un lado relacionados con el conocimiento que tienen los estudiantes sobre conceptos y, por el otro sobre conocimientos de dispositivos TIC.

En la dimensión de manejo saturan ítems como: utilizo Facebook y manejo herramientas para la formación, manejo herramientas ofimáticas, manejo herramientas de edición, manejo herramientas de trabajo colaborativo, manejo herramientas de búsqueda y documentación, manejo cámara fotos digital, manejo lector e-Book, manejo cámara vídeo digital, manejo Tablet y manejo Smartphone; aunque también satura el ítem manejo herramientas organización del tiempo, que versa sobre el uso de herramientas.

Al analizar la dimensión de actitud, con el mismo método de componentes principales, se incluyen los ítems: TIC ayudarán economizar esfuerzo/trabajo profesión, TIC medio útil para seguir formándome, TIC ayudarán economizar esfuerzo/trabajo académico, necesario TIC para desarrollo profesional, TIC necesarias/útiles para mi futuro profesional y Educador profesional competente en TIC. Curiosamente, el ítem sé lo que son las TIC, que no consideramos que debiera incluirse en este factor, satura también en el mismo.

Hay ítems que no se encuentran en la dimensión prevista de las tres establecidas: conocimiento, manejo y actitud. Y se obtienen los siguientes datos en el análisis (tablas 1, 2 y 3 ).

Tabla 1.

Algunos de los ítems no adquieren el peso suficiente (<40) dentro del factor. Otros, sin embargo, son ubicados 
en un factor distinto al que establecían tanto los investigadores como el comité de expertos que validó el contenido del mismo.

La mayoría de los ítems tienen una importante carga o valores altos a la hora de interpretar el factor, lo que nos lleva a considerarlos como determinantes para el mismo.

$\mathrm{Si}$ bien desconocemos el criterio de agrupación, encontramos una aparente relación entre ítems de una misma dimensión, lo que nos lleva a pensar que pueda deberse al desconocimiento de lo preguntado. Este es un hecho reconocido por algunos autores como Ferrando (1996) y Muñiz (1998).

Finalmente se realizó un análisis de fiabilidad en el que se constató su elevada fiabilidad como consistencia interna por medio del estadístico $\alpha$ de Cronbach tanto para cada una de las dimensiones $(\alpha$ conocimiento $=0.92$, $\alpha$ manejo $=0.83$ y $\alpha$ actitud $=0.91)$ como para la escala completa $(\alpha=0.91)$. En tres de los casos presenta un nivel de fiabilidad muy bueno $(\alpha>0,90)$ y se observa una excepción en la dimensión de manejo, en la que el nivel desciende ligeramente, pudiendo considerarse igualmente bueno.

\section{Conclusiones y Discusión}

Para mejorar los planes de formación inicial en lo que se refiere a la competencia digital, es necesario contar con información válida y fiable (Flores y Roig, 2016). Teniendo esto en cuenta, hemos pretendido diseñar un instrumento que reúna estas dos caractarísticas para contribuir a obtener dicha información a partir de la autoevaluación que realizan los alumnos universitarios sobre su competencia digital, desglosada ésta en tres constructos: conocimientos sobre TIC; manejo de dispositivos, herramientas y servicios TIC; así como actitudes hacia las mismas. Estos constructos los encontramos en estudios recientes, como el realizado por Mirete, García y Hernández (2015), y muchas investigaciones sobre el tema, abordan alguno o algunos de los mismos (Almerich, Suárez, Belloch y Orellana, 2010; Cabero, 2014; Cabezas, Casillas y Pinto, 2014; Fernández y Torres, 2015; Valdés, Angulo, Nieblas, Zambrano y Arreola, 2012).

Los análisis llevados a cabo en este trabajo confirman el carácter multidimensional de la competencia digital ya señalada en investigaciones previas como la de Cabero (2004). Los resultados obtenidos en el análisis de fiabilidad permiten afirmar que este cuestionario es un instrumento con una consistencia interna apropiada, válido para medir los constructos de conocimiento, manejo y actitud hacia las TIC. De la misma manera, el análisis factorial arroja un número de factores por dimensión adecuado a lo previsto, lo que permite concluir que el cuestionario diseñado se ajusta al modelo propuesto en sus tres dimensiones. Por ello, consideramos que este instrumento constituye una herramienta útil para futuras investigaciones, cuyo objetivo sea el de profundizar en la competencia digital de los estudiantes universitarios.

\section{Referencias}

Almerich, G., Suárez, J.M., Belloch, C. y Orellana, N. (2010). Perfiles del profesorado a partir del conocimiento de los recursos tecnológicos y su relación con el uso que hacen de estas tecnologías. Revista Complutense de Educación, 21(2), 247-269.

Cabero, J. (2004). Formación del profesorado en TIC: el gran caballo de batalla. Comunicación y Pedagogía. Tecnologías y Recursos Didácticos, 195, 27-37.

Cabero, J. (Dir.) (2014). La formación del profesorado en TIC: Modelo TPACK (Conocimiento Tecnológico, Pedagógico y de Contenido). Sevilla: Secretariado de Recursos Audiovisuales y Nuevas Tecnologías, Universidad de Sevilla.

Cabezas, M., Casillas, S. y Pinto, A.M. (2014). Percepción de los alumnos de Educación Primaria de la universidad de Salamanca sobre su competencia digital. EDUTEC, Revista Electrónica de Tecnología Educativa, $48 . \quad$ doi: http://dx.doi.org/10.21556/edutec.2014.48.156

Centeno, G. y Cubo, S. (2013). Evaluación de la competencia digital y las actitudes hacia las TIC del alumnado universitario. RIE, Revista de investigación Educativa, 31(2), 517-536.

Cubo, S. Martín, B. y García, J.L. (Coords) (2011). Métodos de investigación y análisis de datos en ciencias sociales y de la salud. Madrid: Pirámide.

CRUE y REBIUN (2012). Competencias informáticas $e$ informacionales (CI2) en los estudios de grado. Edición revisada y ampliada. Madrid: CRUE -TICREBIUN. Recuperado de: http://www.rebiun.org/competenciadigital/Documents /CI2_en_las_universidades_2011.pdf

Fernández, J.C., Fernández, M.C. y Cebreiro, B. (2016). Desarrollo de un cuestionario de competencias en TIC para profesores de distintos niveles educativos. PíxelBit, Revista de Medios y Educación, 48, 135-148. doi: http://dx.doi.org/10.12795/pixelbit.2016.i48.09

Fernández, J.M. y Torres, J.A. (2015). Actitudes docentes y buenas prácticas con TIC del profesorado de Educación Permanente de Adultos en Andalucía. Revista Complutense de Educación, 26, 33-49. doi: http://dx.doi.org/10.5209/rev_RCED.2015.v26.43812

Flores, C. y Roig, S. (2016). Diseño y validación de una escala de autoevaluación de competencias digitales para estudiantes de Pedagogía. Píxel-Bit, Revista de Medios y Educación, 48, 209-224. doi: http://dx.doi.org/10.12795/pixelbit.2016.i48.

García M.P. y Morillas, L.R. (2011). La planificación de evaluación de competencias en Educación Superior. REIFOP, Revista Electrónica Interuniversitaria de Formación del Profesorado, 14(1), 113-124.

Gavari, E. (2006). El prácticum en el espacio europeo de educación superior. En E. de Lara y J. Quintanal (Coord.), El prácticum en las titulaciones de educación: Reflexiones y experiencias (pp. 83-92). Madrid: Dykinson. 
Martín, D., Sáenz, M., Santiago, R. y Chocarro, E. (2016). Diseño de un instrumento para evaluación diagnóstica de la competencia digital docente: formación flipped classroom. Revista Didáctica, Innovación y Multimedia, DIM, 33. Recuperado de: http://dim.pangea.org/revistaDIM33/revistanew.htm.

Mirete, A.B., García, F.A. y Hernández, F. (2015). Cuestionario para el estudio de la actitud, el conocimiento y el uso de TIC (ACUTIC) en Educación Superior. Estudio de fiabilidad y validez. Revista Interuniversitaria de Formación del Profesorado, 83(29.2), 75-89.

Roblizo, M., Sánchez, M.C. y Cózar, R. (2015). El reto de la competencia digital en los futuros docentes de Infantil, Primaria y Secundaria: los estudiantes de Grado y Máster de Educación ante las TIC. Prisma social, Revista de Ciencias Sociales, 15, 254-295.

Torres, T. y Vidal, M.A. (2015). Percepción de estudiantes y empleadores sobre el desarrollo de competencias digitales en la educación superior. Revista de Educación, 367, 63-90. Recuperado de: https://sede.educacion.gob.es/publiventa/detalle.action ?cod $=20152$

Unión Europea (2007). Competencias clave para el aprendizaje permanente. Un marco de referencia europeo. Luxemburgo: Oficina de Publicaciones Oficiales de las Comunidades Europeas. Recuperado de:

http://www.mecd.gob.es/dctm/ministerio/educacion/m ecu/movilidad-

europa/competenciasclave.pdf?documentId=0901e72 b80685fb1.

Valdés, A., Angulo, J., Nieblas, E. H., Zambrano, L. y Arreola, C. (2012). Actitudes de docentes de secundaria hacia el uso de las TIC. Investigación educativa duranguense, 6(12), 4-10. doi: 10.5209/rev_RCED.2015.v26.43812 